\title{
Universiteit
}

Leiden

The Netherlands

\section{Proteomic patterns in blood of ovarian cancer patients}

Wegdam, W.; Moerland, P.D.; Buist, M.R.; Ver Loren van Themaat, E.; Blijlevens, B.; Kate, F.J.W. ten; ... ; Aerts, J.M.F.G.

\section{Citation}

Wegdam, W., Moerland, P. D., Buist, M. R., Ver Loren van Themaat, E., Blijlevens, B., Kate, F. J. W. ten, ... Aerts, J. M. F. G. (2007). Proteomic patterns in blood of ovarian cancer patients. Ejc Supplements, 5(8), 36. doi:10.1016/S1359-6349(08)70098-3

Version: $\quad$ Not Applicable (or Unknown)

License: $\quad$ Leiden University Non-exclusive license

Downloaded from: https://hdl.handle.net/1887/65485

Note: To cite this publication please use the final published version (if applicable). 
angiogenesis and combinations of serological biomarkers are more likely to be informative.

Methods: Using ELISA technology, we have developed a panel of biomarkers, measured using GCLP, whose signature could act as a surrogate clinical endpoint to evaluate of anti-angiogenesis therapy considered alongside imaging data. A comprehensive panel of biomarkers will be used in trials of anti-angiogenic drugs including VEGF A,C,D, Placental Growth Factor, sKDR, FGF2, HGF, Ang 1/2, Tie 2, IL-8, SDF-1 and PDGF-B.

Results: Within the framework of the current EU legislation regarding the validation of assays used in clinical trials, we have undertaken a program of 'fit for purpose, fast track' method validation of these biomarkers as singleplex ELISAs. Whilst many commercial kits are available, additional validation issues include generation of external quality control samples, assay performance within clinically relevant matrix (parallelism and dilution linearity). In addition, stability of analytes under various storage and transport conditions has been addressed.

Conclusions: This 'fast track' approach is part of a wider serum biomarker analysis strategy which encompasses a comprehensive sample processing, tracking and analysis system, fully audited by our Quality Assurance team within a specialised GCLP laboratory. To discern druginduced change confidently, pilot clinical studies involve a number of predose baseline measurements essential in order to calculate signal to noise. Multiplex technology will reduce blood volumes for these large numbers of biomarkers if GCLP validation can be achieved

\section{P66}

\section{Proteomic patterns in blood of ovarian cancer patients}

W. Wegdam, P.D. Moerland, M.R. Buist, E. Ver Loren van Themaat, B. Blijlevens, F.J.W. ten Kate, H.J.C. Hoefsloot ${ }^{2}$, D.G. Richel, C.G. de Koster, J.M.F.G. Aerts. ${ }^{1}$ Academic Medical Center, Amsterdam, The Netherlands; ${ }^{2}$ University of Amsterdam, The Netherlands

Background: Despite extensive therapy the 5-year survival rate of advanced ovarian carcinoma remains poor. Mass spectrometry can be used to discover new proteins associated with ovarian cancer. Recent studies have indicated that there are still many unresolved problems involving this technique. Our aim is to solve some of these problems and to develop a specific protein profile in serum of patients with ovarian cancer. Methods: For our pilot study 14 patients with newly diagnosed ovarian adenocarcinoma and 14 patients with benign gynecological diseases were included. Blood was collected using a strict protocol whereby samples are frozen and stored at $-80^{\circ} \mathrm{C}$ immediately after collection. The samples were processed on the CM10 and Q10 ProteinChip array using the SELDI-TOF. Pre-processing was done comparing the method implemented in the Ciphergen software to an adapted version of the Cromwell package. After which we assessed the quality of the spectra using various clustering methods. Classification was done using 5 different methods including three, SVM, naiveBayes, DLDA and PCDA, to reliably identify the two sample classes. The classifiers were validated with repeated random sampling methodology.

Results: The different hierarchical clustering methods and subsequent bootstrapping revealed that we could identify potential outliers and that the quality of the remaining spectra was good. On average, 5 peaks are differentially expressed between the two groups with a false discovery rate $<0.05$. The class prediction obtained from the 5 different classification methods on a 1000 random test sets has a mean error rate of $18 \%$ $(95 \% \mathrm{Cl} 0-50 \%)$. Although the Cromwell pre-processing method gave a better estimation of the true $\mathrm{m} / \mathrm{z}$ values compared to the pre-processing done with the Ciphergen software the classification results are similar. Conclusions: These results indicate that it is possible to separate patients from controls with proteomic data and a variety of pre-processing and classification methods.
P21

Serum insulin-like growth factor-I and insulin-like growth factor binding protein-3 are not useful markers of prostate cancer

M. Zancan ${ }^{1}$, R. Dittadi ${ }^{2}$, V. Scattoni ${ }^{3}$, A. Fandella ${ }^{4}$, P. Del Bianco ${ }^{5}$, M. Gion ${ }^{6} .{ }^{1}$ Association ABO for the Application of Biotechnologies in Oncology clo Centre for the Study of Biological Markers of Malignancy-IOV IRCCS Regional Hospital, AULSS12, Venice, Italy;

${ }^{2}$ Centre for the Study of Biological Markers of Malignancy-IOV Veneto IRCCS Regional Hospital, AULSS12, Venice, Italy; ${ }^{3}$ Urology Department, University Vita Salute, S. Raffaele Hospital, Milan, Italy; ${ }^{4}$ Urology Department, Treviso Hospital, Italy; ${ }^{5}$ Clinical Trials and Biostatistics Unit Istituto Oncologico Veneto, IRCCSS, Padova, Italy; ${ }^{6}$ Centre for the Study of Biological Markers of malignancy-consortium Istituto Oncologico Veneto IRCCS, Regional Hospital, AULSS12, Venice, Italy

Background: Prostate cancer (PCA) is the most common cancer in men in Western countries, with a significant increasing incidence. Although PSA is a well known biomarker for prostate cancer, its diagnostic specificity in detecting PCA from other benign prostate diseases such as benign prostatic hyperplasia (BPH), is still debated. Several studies reported that PCA patients present high levels of IGF-I and IGFBP-3.

The aim of the present study was to assess if the value of these biomarkers in combination with PSA (Total or Free/Total ratio) could improve humoral diagnostic performance in patients with PCA.

Methods: Two hundred eighty patients were included in the study. The patients were enrolled by three General Hospitals (Venezia, Milan and Treviso), between March 1996 and October 1999. 177 patients were diagnosed with BPH (mean age 67 years; range 44-88 years) and 103 diagnosed with PCA (mean age 67 years; range 44-88 years) according to clinical and pathological criteria. IGF-I and IGFBP-3 were measured employing two commercially available immunoassays; RIA (Biosource/Medgenix) and IRMA (DSL, Inc, Webster, Texas) respectively. Total and Free PSA were measured using an automated instrument (ADVIA-Centaur, Bayer). Statistical analyses were conducted using a commercial available software.

Results: PCA patients had significantly higher Free and Total PSA values than BPH patients $(p \leqslant 0.0001)$ and significantly lower Free PSA/Total PSA ratio levels. In the present study we found a significant high positive correlation between IGF-1 and IGFBP-3 ( $r=0.72, p<0.0001)$, but the serum concentration of IGF-I and IGFBP-3 did not significantly differ between patients with PCA and $\mathrm{BPH}$ neither considering their absolute levels or their ratio $(p=0.5952)$. Free and Total PSA levels did not correlate with IGF-1 and IGFBP-3 levels, neither in PCA patients nor in $\mathrm{BPH}$ patients. Receivers Operating Curve (ROC) analysis matching PSA (total or free/total ratio) with IGF-1 or IGFBP-3 did not show any significant advantages, when compared to the use of PSA (Total or Free/Total ratio) alone.

Conclusions: The present study, according to other reports, did not confirm the usefulness of IGF-1 and IGF binding protein-3, in prostate cancer. Total PSA or Free PSA//Total PSA is until now the most reliable humoral tool in the diagnosis of prostate cancer. 